\title{
Correspondence
}

\section{An Unfounded Conclusion from a Confounded Study}

To the Editor: In the recent article by Edmonds and Vinson, "Three Measures of Sleep, Sleepiness, and Sleep Deprivation and the Risk of Injury: A Case-Control and Case-Crossover Study," ${ }^{1}$ the correlative relationships between self-reported sleepiness, sleep quality, and sleep amount with risk of accident-related injury are reported. The "major conclusion" is that "sleepiness and sleep deprivation, at least as they are commonly experienced, are weakly and inconsistently associated with injury risk."

However, this conclusion is itself only weakly and inconsistently supported by the study findings: For example, the authors indicate that in their "multifaceted analyses of sleepiness and sleep deprivation, [there was] little evidence for association between [self-reported] sleep and injury," despite also reporting that better recent sleep quality was associated with lower risk of injury. Similarly, based on a negative correlation between selfreported sleepiness and injury rate, the authors surmise that feeling sleepy might prompt behavioral changes that serve to reduce injury risk (ie, providing a "protective effect"). However, they also indicate that controlling for "location and activity" (the only 2 relevant aspects of behavior reported in the paper) had "little effect."

Unfortunately, it would be difficult to draw conclusions from this study even if the findings had been consistent. This is because (as the authors themselves discuss) the results were not only subject to the considerable problems typically associated with retrospective self-report data, but the study was confounded: Injured subjects were interviewed in the hospital, under conditions that could reasonably be considered relatively stressful (eg, emotional turmoil associated with the recent accident), whereas control group subjects were interviewed by telephone (presumably at home, where they were less likely to be distressed). Compounding these problems, the authors failed to use any of the several available validated measures of subjective sleepiness (eg, Stanford Sleepiness Scale) ${ }^{2}$ and sleep quality (eg, The Pittsburgh Sleep Quality Index), ${ }^{3}$ making it difficult to compare their results with those of prior field and laboratory studies.

Given the inconsistent findings, failure to use validated measures, and the confounding in this study, and especially given the fact that the authors were apparently aware of these problems (they acknowledge in the discussion section that the their results "might be due to confounding or reporting bias"), it was overstated to conclude that sleepiness may only weakly increase risk. Similarly, given the potential implications, the conjecture that sleepiness actually provides a "protective effect" against accidents by promoting more cautious behavior is not only unsupported by their data, it is contrary to the weight of scientific evidence. ${ }^{4}$
Even if their study had been well controlled, used validated measures of sleepiness, and produced consistent results, a more carefully considered conclusion would have been warranted. For example, the conclusion that "people involved in accidents resulting in injury are less likely to report having been sleepy at the time of the accident" would leave open the possibility that the injured subjects actually had been less sleepy, but also invites other, more plausible interpretations (eg, people involved in accidents resulting in injury are less willing to admit to sleepiness).

Tracy L. Rupp

Thomas J. Balkin

Walter Reed Army Institute of Research, Silver Spring, MD

Disclaimer: The views expressed in this letter are those of the authors and do not reflect the official policy or position of the Walter Reed Army Institute of Research, the Department of the Army, the Department of Defense, the U.S. Government, or any institutions with which the authors are affiliated.

\section{References}

1. Edmonds J, Vinson DC. Three measures of sleep, sleepiness, and sleep deprivation and the risk of injury: a casecontrol and case-crossover study. J Am Board Fam Med 2007;20:16-22.

2. Hoddes E, Zarcone V, Smythe H, Phillips R, Dement, WC. Quantification of sleepiness: a new approach. Psychophysiology 1973;10:431-6.

3. Buysse DJ, Reynolds CF, Monk TH, Berman SR, Kupfer DJ. The Pittsburgh Sleep Quality Index: a new instrument for psychiatric practice and research. Psychiatry Res 1989; 28:193-213.

4. Dinges DF. An overview of sleepiness and accidents. J Sleep Res 1995;4 Suppl 2:4-14.

doi: 10.3122/jabfm.2007.04.070061

The above letter was referred to the authors of the article in question, who offer the following reply.

\section{Judging Quality of Rural Healthcare}

To the Editor: I appreciate the critique from Drs. Rupp and Balkin. Our findings are indeed counterintuitive, and open debate will hopefully lead to clearer understanding.

We used the Positive Affect Negative Affect Scale because it covers a range of emotional states. As noted in our paper, it has been validated. ${ }^{1,2}$ It has not been widely used in sleep research, but we were also interested in anger $^{3}$ and other emotions.

We compared injured people not only to an uninjured control group, but also to themselves 24 hours before. Granted, recall bias is still likely, but the very similar findings with 2 separate control groups enhance the credibility of the results.

We all think that sleepiness and sleep deprivation are strongly associated with injury risk, but look again at the 